\title{
Medial Collateral Ligament Release Facilitates Exposure in Revision Total Knee Arthroplasty
}

\author{
Jad Bou Monsef, MD ${ }^{1} \quad$ Friedrich Boettner, MD ${ }^{1}$ \\ ${ }^{1}$ Adult Reconstruction and Joint Replacement Division, Hospital for \\ Special Surgery, New York, New York \\ J Knee Surg Rep 2015;1:39-43.
}

\author{
Address for correspondence Jad Bou Monsef, MD, Adult \\ Reconstruction and Joint Replacement Division, Hospital for Special \\ Surgery, 535 East 70th Street, New York, NY 10021 \\ (e-mail: jadnbm@gmail.com).
}

\begin{abstract}
Keywords

- exposure

- revision total knee arthroplasty

- orthopedic surgery

- knee arthroplasty

Incidence of revision total knee arthroplasty is growing. Revision surgery aims at preserving the collateral ligaments to minimize implant constraint and stress on the implant-cement-bone interface. Exposure in the stiff knee can be challenging as a result of loss of soft tissue elasticity and capsular scarring brought on by multiple surgeries. If a quadriceps snip does not provide adequate visualization a quadriceps turndown or a tibial tubercle osteotomy is recommended to improve exposure. Such extensile approaches have various limitations and might risk compromising the extensor mechanism, recovery time, bone fixation, or wound healing. The current case report describes a novel technique to combine a quadriceps snip with cutting the medial collateral ligament to gain exposure. While patients require a hinged implant, the integrity of the extensor mechanism is preserved. This allows for immediate active and passive range of motion and places this approach at an advantage over a quadriceps turndown or tibial tubercle osteotomy.
\end{abstract}

Exposure in revision knee replacement can be challenging. Following the medial parapatellar incision, ${ }^{1}$ the first step in every revision surgery is a sufficient medial capsule release on the tibia and external rotation of the tibia to relieve tension on the extensor mechanism. Additional surgical options have been described to improve exposure. The quadriceps snip or rectus snip is usually the first step as it entails minimal risk to the extensor mechanism, and allows nearly identical postoperative rehabilitation and outcome. ${ }^{2}$ The oblique extension of the arthrotomy at 45 degrees superiorly and laterally is parallel to the fibers of the vastus lateralis and preserves its musculotendinous junction. This facilitates eversion and displacement of the patella and extensor mechanism. ${ }^{3}$ In fact, a quadriceps snip combined with a subperiosteal medial collateral ligament $(\mathrm{MCL})$ release provides adequate exposure for most revision total knee arthroplasty (TKA). ${ }^{4}$

In extensively scarred or ankylosed knees, a full quadriceps release might be necessary. This quadriceps turndown, an incision connecting the medial arthrotomy with a lateral retinaculum release, provides wide exposure at the expense of a weakened extensor mechanism as well as restricted postoperative rehabilitation. ${ }^{5}$

Alternatively, a tibial tubercle osteotomy can be utilized to facilitate exposure and tibial component removal. Although it spares the quadriceps and provides excellent exposure, ${ }^{6}$ risks include nonunion, drainage from the area leading to sinus tract formation, the potential for deep space infection, ${ }^{5}$ and the potential of tibial fracture. ${ }^{7}$

Wide exposure is crucial to allow component removal, bone reconstruction, and reimplantation while reducing operative time and risks. We describe a novel technique used with two patients to gain exposure for revision and implantation of rotating hinge knee prosthesis. At 2-year follow-up, the patient walked painlessly, without the use of assisting devices and had a postoperative range of motion of 0 to $110-110$ degrees. received

March 26, 2013

accepted after revision

February 8, 2015

published online

May 27, 2015
DOI http://dx.doi.org/

10.1055/s-0035-1551546. ISSN 2326-2729.
Copyright $\odot 2015$ by Thieme Medical Publishers, Inc., 333 Seventh Avenue, New York, NY 10001, USA. Tel: +1(212) 584-4662.
License terms

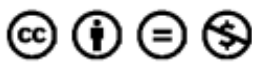




\section{Case 1}

An 80-year-old male patient, underwent bilateral TKAs in 1996 at an outside institution. He had done fairly well until 2005, when he was diagnosed with an expansile lytic lesion in the right proximal fibula. Increased activity in the proximal and lateral right tibia was seen on a bone scan, followed by excision of the mass that revealed fibrous tissue with degenerate material from the prosthesis. In 2007, recurrence of the mass in addition to another medial distal femur mass led to a foot drop, and the patient underwent excision of both masses as well as resection of the fibular head and decompression of the peroneal nerve at the same institution. Pathology showed reactive xanthogranulomatous pseudotumors, an osteolytic reaction. ${ }^{8}$ A month later he had an incision and drainage of an infected seroma with intraoperative cultures growing coagulase-negative Staphylococcus and Corynebacterium species. He received 2 weeks of levofloxacin and had a wound vac machine for 4 months. When he presented to the senior author (F.B.) in February 2008, he had lost $15 \mathrm{~kg}$, the wound was erythematous and still draining. Plain films showed erosion and secondary heterotopic calcifications suggestive of deep implant infection (-Figs. 1 and 2). The wound culture revealed methicillin-sensitive Staphylococcus aureus. Magnetic resonance imaging (MRI) and X-ray revealed a septic joint with osteomyelitis and osteolysis, gas densities in the

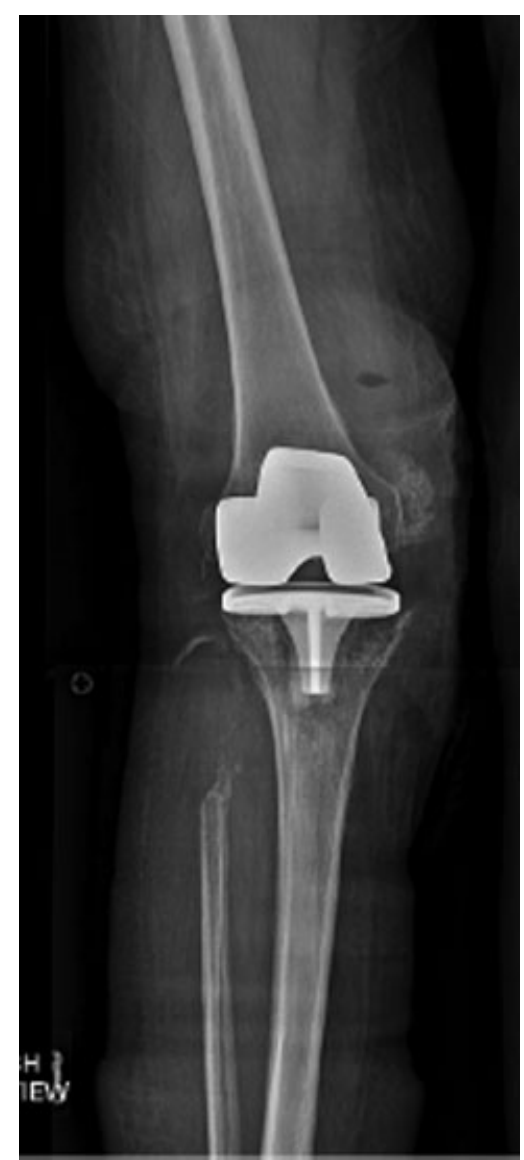

Fig. 1 Preoperative anteroposterior radiograph.

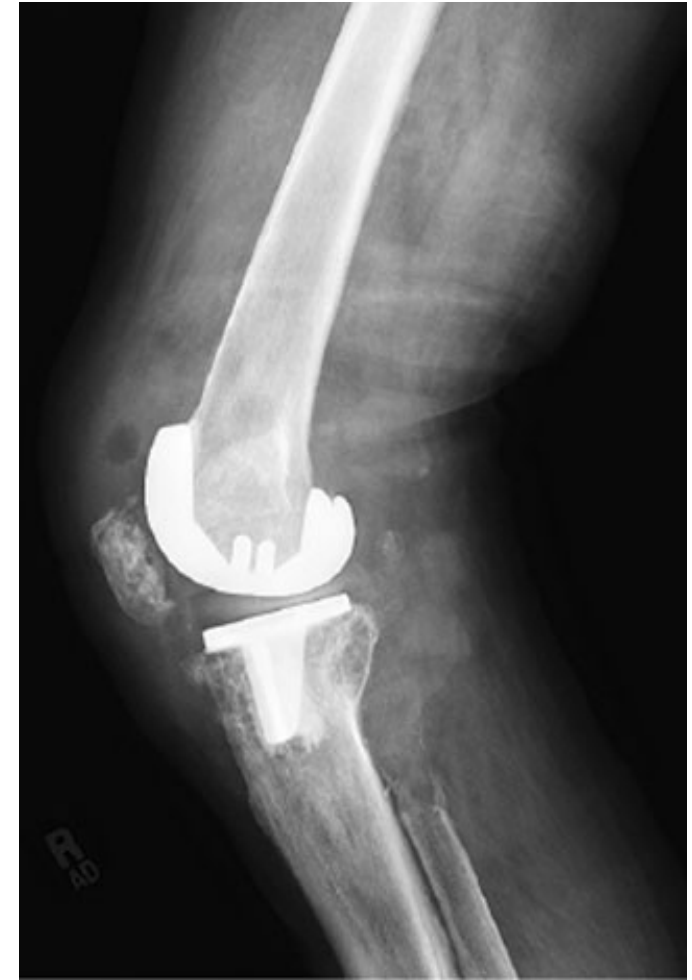

Fig. 2 Preoperative lateral radiograph.

suprapatellar bursa, and an abscess in the medial soft tissues of the knee (-Figs. 3 and $\mathbf{4}$ ). At the time of the initial presentation the patient had a Western Ontario and McMaster Osteoarthritis Index (WOMAC) score of 21, with a right knee range of motion from 15 degrees flexion contracture to 45 degrees flexion. Joint fluid analysis showed 36,750 WBC/

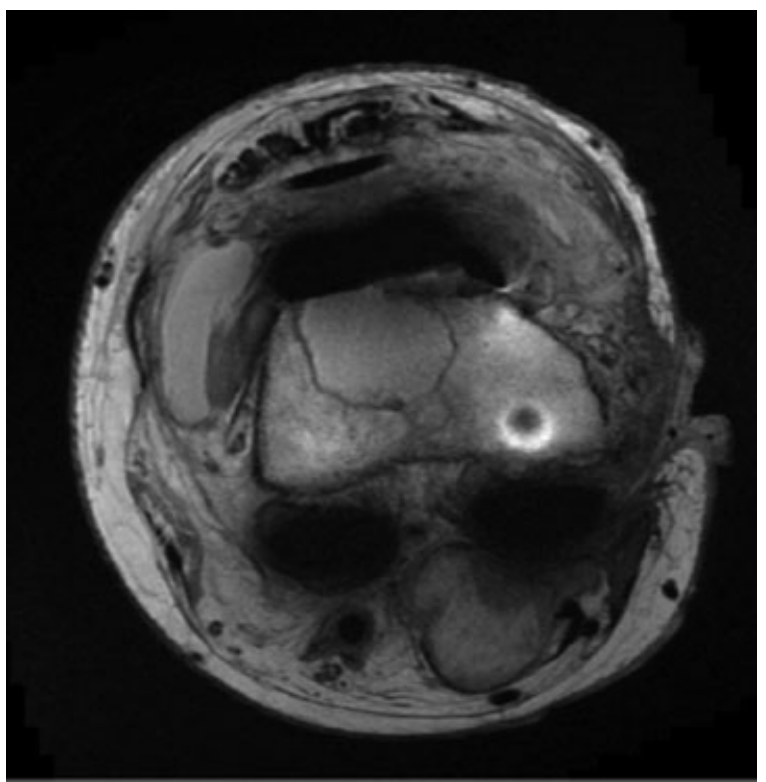

Fig. 3 Preoperative MRI showing joint effusion, a draining fistula, and an abscess. MRI, magnetic resonance imaging. 


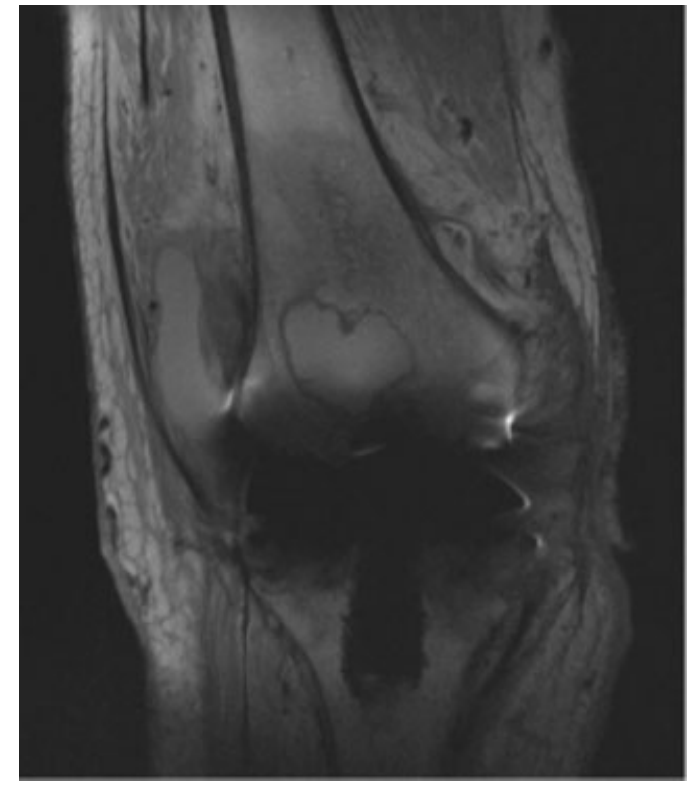

Fig. 4 Preoperative MRI, coronal. MRI, magnetic resonance imaging.

$\mathrm{mL}$ as well as Enterococcus and Staphylococcus sensitive to most antibiotics.

\section{The Procedure}

The first stage of the procedure entailed explantation of the infected prosthesis. After a median parapatellar approach, a medial subperiosteal tibial release was followed by scar tissue excision in the medial and lateral gutters. Despite a quadriceps snip adequate exposure of the femoral and tibial component was not achieved. By performing a medial release around the distal medial femoral epicondyle the MCL was now released. Releasing the medial soft tissue envelope made it possible to apply valgus stress and expose the femoral component. In other words the knee was "bent" in the coronal plane (valgus stress) to avoid any tension on the extensor mechanism. An antibiotic-containing static spacer was inserted and the patient was treated for 6 weeks with intravenous (IV) Zosyn (Baxter Healthcare Corp. Deerfield, IL) and daptomycin through a PICC line.

The second stage of the revision took place 5 months after explantation, after reaspiration of the joint was negative, and an MRI confirmed adequate debridement of soft tissue without residual infection and the patient underwent a DePuy SROM rotating hinge prosthesis (DePuy Synthes Joint Reconstruction, Warsaw, IN). Two years after the surgery, the patient ambulates painlessly with a cane, achieves 100 degrees of knee flexion and full extension (-Figs. 5 and $\mathbf{6}$ ).

\section{Case 2}

A 59-year-old hypertensive, diabetic, asthmatic female patient, with Osler-Weber-Rendu syndrome and chronic anemia was referred to our clinic. She had been transfusiondependent through a Groshong catheter, which was repeatedly infected and changed. She underwent a primary left TKA in 1994, and subsequent patellar resurfacing in 2004 in an

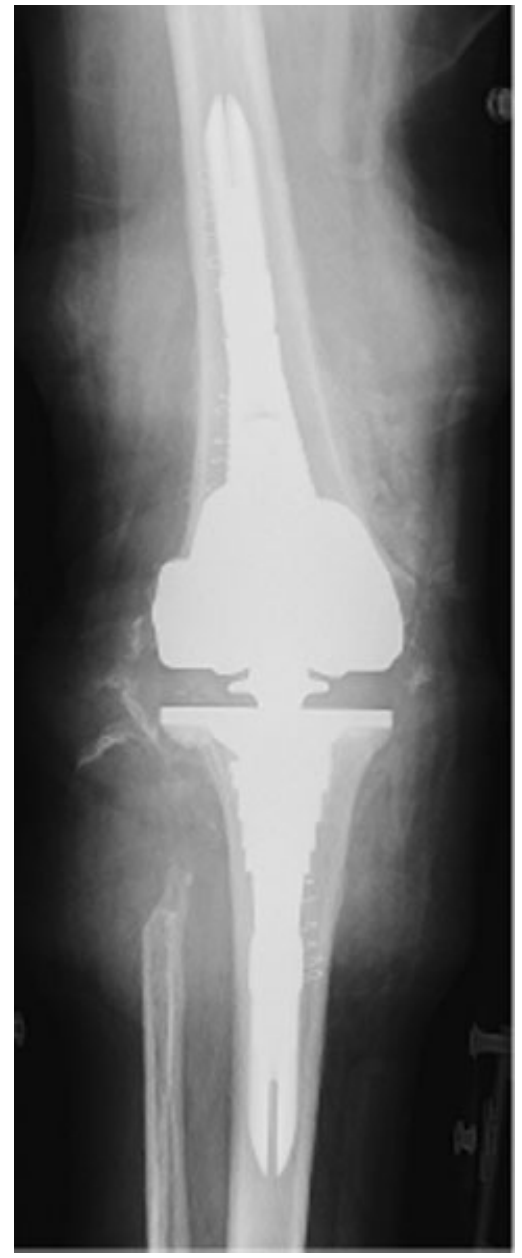

Fig. 5 Postoperative anteroposterior radiograph.

outside hospital. She subsequently developed recurrent Staphylococcus epidermidis infections that lead to two consecutive two-stage revisions in 2 years. She was treated with a prolonged course of antibiotics each time. A few months after the last surgery, she was hospitalized for sepsis secondary to an infected PICC line, which was then removed. During that time she developed severe pain with weight-bearing, associated with significant left knee swelling and a synovial culture growing S. epidermidis. Repeat culture after finishing a course of antibiotics was negative, but X-ray and leukocyte scan suggested focal areas osteomyelitis as well as the possible loosening of the tibial component. She presented our office several months later with persistent knee pain and stiffness. Her range of motion in the left knee was 0 to 25 degrees of flexion.

\section{The Procedure}

The initial attempt to expose the components included: medial parapatella approach, medial capsular release, scar tissue excision out of the infrapatellar and suprapatellar gutter and a quadriceps snip. Because of the stiffness of the extensor mechanism flexion and implant exposure was not achieved with these steps. By performing a medial release around the distal medial femoral epicondyle the MCL was 


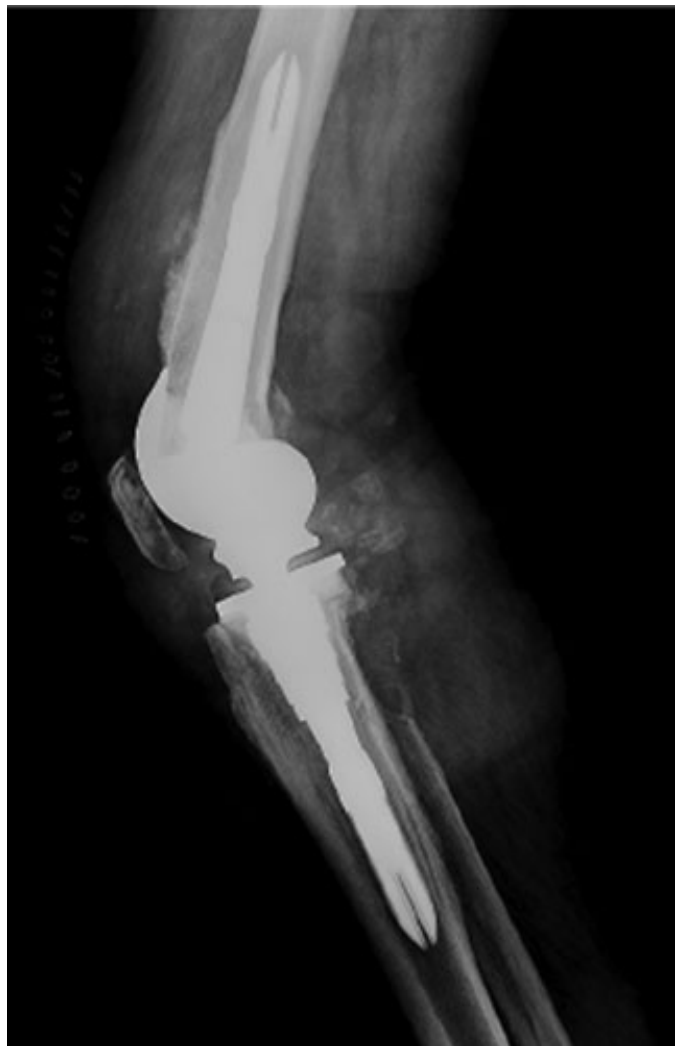

Fig. 6 Postoperative lateral radiograph.

now released. Releasing the medial soft tissue envelope made it possible to apply valgus stress and expose the femoral component. In other words, the knee was "bent" in the coronal plane (valgus stress) to avoid any tension on the extensor mechanism. The components were removed and a static antibiotic-containing cement spacer was inserted. Intraoperative cultures were positive for $S$. epiderdimis. The patient completed 8 weeks of IV antibiotics through a PICC line. She was then cleared for reimplantation with a hingedknee prosthesis. At the time of reimplantation, the knee was exposed with a quadriceps snip and MCL release only.

At 2-year follow-up, the patient ambulated painlessly using a cane and demonstrated a range of motion of 0 to 110 degrees.

\section{Discussion}

Earlier generation hinged implants were associated with suboptimal clinical outcomes and high failure rates, ${ }^{9}$ which was attributed to the design features causing excessive shear stresses and particulate wear. ${ }^{10}$ Advances in material science and design engineering lead to the rotational hinged systems. Many studies reveal promising short-term outcome of the new generation implants such as the DePuy S-ROM, successfully achieving pain relief and joint stability in complex cases. $^{10,11}$ The hinge substitutes for deficiency or complete lack of ligamentous and soft tissue knee support. Potential indications for such implants include MCL disruption, massive bone loss of the distal femur or proximal tibia (including

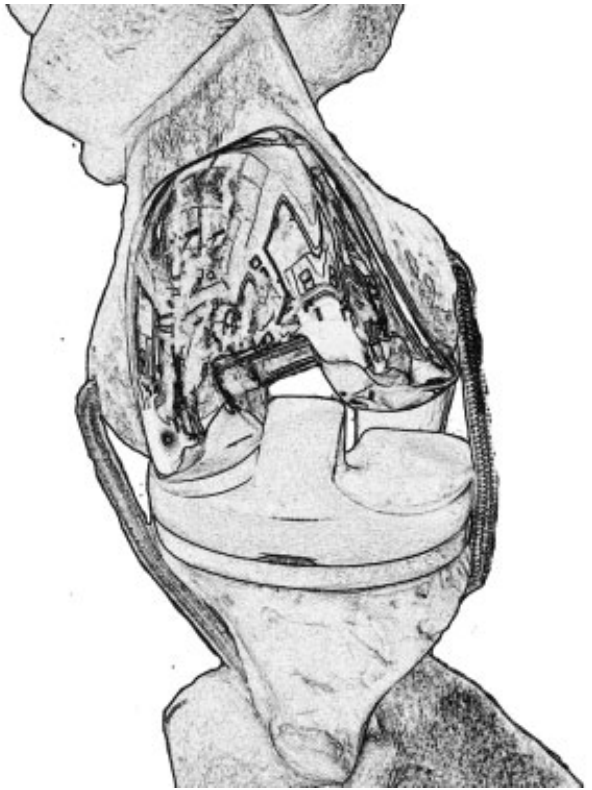

Fig. 7 Exposure with valgus stress with $M C L$ intact. $M C L$, medial collateral ligament.

collateral ligament origin or insertion), extensor mechanism disruption requiring reconstruction in an unstable knee, ankylosis requiring a femoral peel exposure with moderate or severe residual flexion extension gap imbalance, ${ }^{12}$ and a salvage procedure in low mobility patients. ${ }^{13,14}$

The risk of rupture or avulsion of the patellar tendon while attempting exposure is consequently higher in revision arthroplasty, especially in stiff knees with less than 75 degrees of range of motion. The weakest point being the insertion at the tibial tubercle, intraoperative avulsion represents a potentially catastrophic complication. Direct suture repair has shown unfavorable failure rates, and repair with augmentation necessitates immobilizing an already stiff and impaired knee, jeopardizing the outcome of revision surgery. ${ }^{5}$

Adequate exposure is paramount for successful knee reconstruction. ${ }^{5}$ In situations where a rotating hinge knee is implanted, the collateral ligaments are expandable. Transection of the MCL with subsequent external rotation and valgus stress on the tibia as performed in the described cases is a simple technique to facilitate exposure in revision total knee replacement. This technique allows successful revision surgery without resorting to other extensile maneuvers that increase the flexion angle at the expense of the extensor mechanism (-Figs. 7 and 8). In two-stage procedures for infection, the MCL release for knee explantation might not impact implant selection since 6 weeks in a static spacer after the procedure often result in the healing of the MCL release. Since this approach does not compromise the integrity of the extensor mechanism, it has significant benefits for early mobilization and ROM exercises. This technique can be applied to the very stiff knee undergoing primary total knee replacement or a takedown of knee fusion, however, additional implant constrain to compensate for the MCL insufficiency is necessary. The senior author (F.B.) has not 


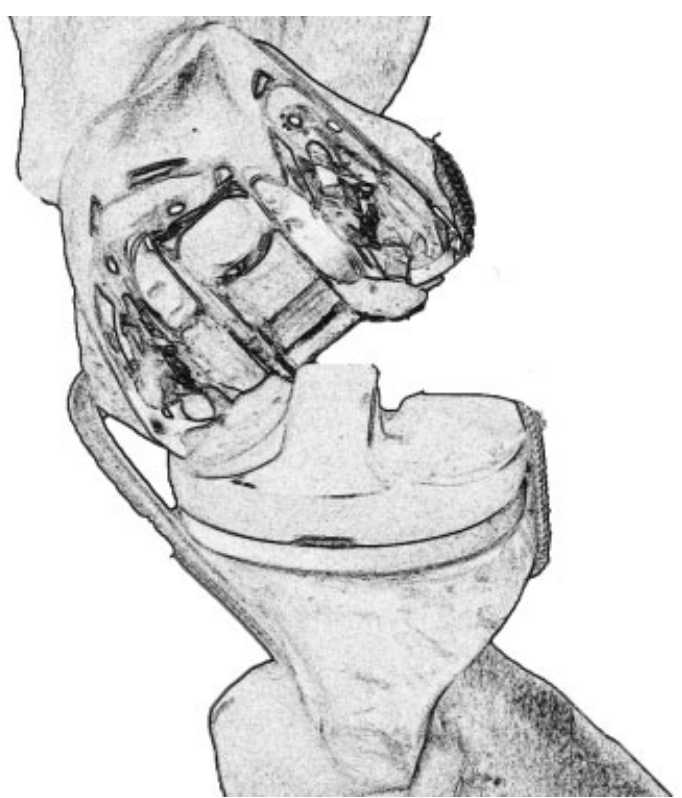

Fig. 8 Increased exposure after MCL release. MCL, medial collateral ligament.

yet encountered the need to apply this technique in primary total knee replacement.

\section{Conclusion}

The current article describes a new technique to expose the stiff knee by releasing the MCL as part of a periosteal soft tissue release around the distal medial femoral condyle and "flexing" the knee in the coronal plane with valgus stress.

\section{References}

1 Barrack RL, Smith P, Munn B, Engh G, Rorabeck C. The Ranawat Award. Comparison of surgical approaches in total knee arthroplasty. Clin Orthop Relat Res 1998;(356):16-21

2 Meek RM, Greidanus NV, McGraw RW, Masri BA. The extensile rectus snip exposure in revision of total knee arthroplasty. J Bone Joint Surg Br 2003;85(8):1120-1122

3 Younger AS, Duncan CP, Masri BA. Surgical exposures in revision total knee arthroplasty. J Am Acad Orthop Surg 1998;6(1): 55-64

4 Jacofsky DJ, Della Valle CJ, Meneghini RM, Sporer SM, Cercek RM; American Academy of Orthopaedic Surgeons. Revision total knee arthroplasty: what the practicing orthopaedic surgeon needs to know. J Bone Joint Surg Am 2010;92(5):1282-1292

5 Engh GA. Exposure options for revision total knee arthroplasty. In: Bono JV, Scott RD, eds. Revision Total Knee Arthroplasty. New York, NY: Springer; 2005:63-75

6 Whiteside LA, Ohl MD. Tibial tubercle osteotomy for exposure of the difficult total knee arthroplasty. Clin Orthop Relat Res 1990; (260):6-9

7 Whiteside LA. Exposure in difficult total knee arthroplasty using tibial tubercle osteotomy. Clin Orthop Relat Res 1995;(321):32-35

8 Jones LC, Hungerford DS. Cement disease. Clin Orthop Relat Res 1987;(225):192-206

9 Rand JA, Chao EY, Stauffer RN. Kinematic rotating-hinge total knee arthroplasty. J Bone Joint Surg Am 1987;69(4):489-497

10 Jones RE, Skedros JG, Chan AJ, Beauchamp DH, Harkins PC. Total knee arthroplasty using the S-ROM mobile-bearing hinge prosthesis. J Arthroplasty 2001;16(3):279-287

11 Barrack RL. Evolution of the rotating hinge for complex total knee arthroplasty. Clin Orthop Relat Res 2001;(392):292-299

12 Deehan DJ, Murray J, Birdsall PD, Holland JP, Pinder IM. The role of the rotating hinge prosthesis in the salvage arthroplasty setting. J Arthroplasty 2008;23(5):683-688

13 Pour AE, Parvizi J, Slenker N, Purtill JJ, Sharkey PF. Rotating hinged total knee replacement: use with caution. J Bone Joint Surg Am 2007;89(8):1735-1741

14 Hernández-Vaquero D, Sandoval-García MA. Hinged total knee arthroplasty in the presence of ligamentous deficiency. Clin Orthop Relat Res 2010;468(5):1248-1253 